\title{
Tessellation and Visibility Representations of Maps on the Torus*
}

\author{
B. Mohar ${ }^{1}$ and P. Rosenstiehl ${ }^{2}$ \\ ${ }^{1}$ Department of Mathematics, University of Ljubljana, Slovenia \\ ${ }^{2}$ Centre d'Analyse et de Mathématique Sociales, EHESS, Paris, France
}

\begin{abstract}
The model of the torus as a parallelogram in the plane with opposite sides identified enables us to define two families of parallel lines and to tessellate the torus, then to associate to each tessellation a toroidal map with an upward drawing. It is proved that a toroidal map has a tessellation representation if and only if its universal cover is 2-connected. Those graphs that admit such an embedding in the torus are characterized.
\end{abstract}

\section{Introduction}

Given a graph $G$, let $V(G)$ be the set of vertices of $G, E(G)$ the set of edges of $G$. For $A \subseteq V(G)$ we denote by $E(A)$ the set of edges of $G$ with both ends in $A$.

A map $M$ on a surface $\Sigma$ is a connected graph $G$ together with a 2-cell embedding of $G$ in $\Sigma$. Two maps are equivalent if there is a homeomorphism of $\Sigma$ mapping the graph of the first map onto the graph of the second. It is well known that the equivalence classes of maps on orientable surfaces correspond naturally to rotation systems on the underlying graphs [4]. Let us recall that a rotation system on a graph $G$ is a set of cyclic permutations $\pi_{v}(v \in V(G))$ where $\pi_{v}$ cyclically permutes edges emanating from $v$; $\pi_{v}$ corresponds to the cyclic order of these edges around $v$ on the surface. A similar combinatorial representation can be given for maps on nonorientable surfaces (see, e.g., [4] or [10]). The rotation system around the faces of $M$ defines the dual map $M^{*}$. A map $M$ and its dual $M^{*}$ can be simultaneously drawn in $\Sigma$ such that each vertex of $M^{*}$ corresponds to an interior point of the corresponding face of $M$ (and vice versa), and such that precisely dual pairs of edges cross each other.

\footnotetext{
* The first author was supported in part by the Ministry of Science and Technology of Slovenia, Research Project P1-0210-101-94. The second author's work was partially supported by the ESPRIT Basic Research Action No. 7141 (ALCOM II).
} 


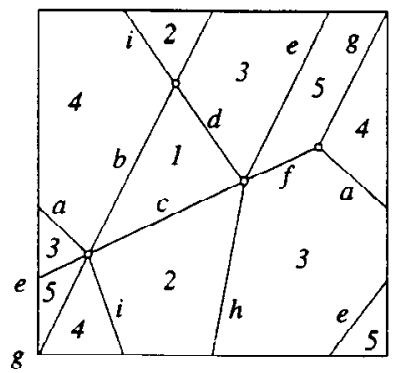

(a)

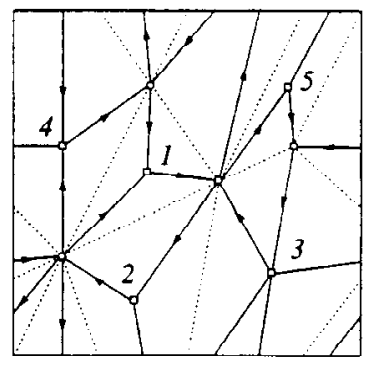

(b)

Fig. 1. A toroidal map and its angle map.

If $M$ is a map in $\Sigma$ whose underlying graph has $n$ vertices, $e$ edges, and $f$ faces, then $n-e+f=\chi(\Sigma)$ where $\chi(\Sigma)$ is the Euler characteristic of the surface $\Sigma$.

In this paper we only consider maps on closed surfaces (i.e., compact surfaces without boundary) and maps in the plane. Most attention is given to the torus which can be represented by a parallelogram $Q$ in the plane whose opposite sides are pairwise identified. This representation of the torus carries the local geometry of the plane and hence we call it the flat torus.

From now on we will assume that $\Sigma$ is a closed surface, $M$ a map on $\Sigma$, and $G$ the underlying graph of $M$. An angle of $M$ (resp., $M^{*}$ ) is a pair of consecutive arcs at a vertex $v$ of $M$ (resp., a face $f$ of $M$ ). The angle map of $M$ is a map $\mathcal{A}$ on $\Sigma$ whose vertices are the vertices of $M$ plus the vertices of $M^{*}$ (i.e., the faces of $M$ ), and whose edges are the angles of $M$, each angle being incident with the corresponding vertex and face of $M$ [12]. The set of angles incident with a vertex $v$ of $M$ has a local rotation determined by $M$, and the set of angles incident with a face of $M$ inherits the local rotation from $M^{*}$. The angle map $\mathcal{A}$ is bipartite and each face of $\mathcal{A}$ is a quadrangle whose diagonals are a pair of dual edges of $M$ and $M^{*}$. An example is shown in Fig. 1(b) where the dotted lines represent the edges of $M$ and they are not part of $\mathcal{A}$. The dual map of $\mathcal{A}$ is known as the medial map of $M$.

An important concept related to drawings of graphs in the plane is the notion of upward drawings where each edge of a graph is oriented and drawn in the plane $\mathbf{R}^{2}$ so that the ordinate monotonically increases when we traverse the edge according to its orientation. In order to define an upward drawing of a graph $G$ on the torus, we first need a definition of a monotone arc. Consider, without loss of generality, the flat torus obtained from a parallelogram $Q$ in the plane. The lines parallel to the sides of $Q$ oriented according to the usual axes determine horizontal and vertical circuits on the torus. At each point of the torus two circuits cross, the vertical one being crossed always by the horizontal one, for instance, from left to right. A (polygonal) arc on the torus is monotone if it can be oriented such that by traversing the arc in the chosen direction, one crosses horizontal circles only from bottom to top. In particular, the vertical circuits are examples of monotone arcs. Let us observe that a monotone arc may cross a horizontal circuit more than once by "winding" around two or more times. The definition of horizontal circles is easily extended to the case when the horizontal direction is not necessarily parallel to 
a side of $Q$. Monotone arcs are defined accordingly. An upward drawing of a toroidal map $M$ is a map equivalent to $M$ drawn on the torus with monotone (polygonal) arcs such that at each vertex $v$ of $M$, at least one edge incident to $v$ enters $v$ from below (with respect to the chosen horizontal direction) and at least one edge enters $v$ from above (see Fig. 1(a)). The authors have shown in [9] by using network flow techniques that every toroidal map with 2-connected universal cover admits a drawing in the flat torus that is an upward drawing and, moreover, the corresponding drawing of the dual map is upward at the same time (with respect to the vertical circuits instead of the horizontal ones).

A stronger concept than upward drawings is the tessellation representation of a map. This concept was investigated in case of planar graphs by Tamassia and Tollis [14]. Given horizontal and vertical directions $\Delta$ and $\Delta^{\prime}$ of the flat torus (possibly distinct from the directions of the sides of the fundamental parallelogram $Q$ ), we consider a family of horizontal line segments called the vertices, and a family of vertical line segments called the faces, all disjoint except that each segment of one family touches at each end a segment of the other family at one of its internal points. A map is associated with a tessellation in the following way. Horizontal segments are the vertices, vertical segments the faces. Each of the obtained quadrangular regions determines an edge of the map; it is, by definition, incident with two vertices by its two horizontal sides, and with two faces by its two vertical sides. The local rotation $\pi_{v}$ around the vertex $v$ is determined by the order of edges obtained by traversing the boundary of a small neighborhood of the vertical segment corresponding to $v$ in the clockwise direction. This rule determines the map up to equivalence. A tessellation of the torus is then a representation of a toroidal map defined by its vertices, faces, and edges as introduced above. An example of a tessellation and the corresponding map is shown in Fig. 2 and a tessellation representation of the map from Fig. 1(a) is presented in Fig. 3. Our main result is that a toroidal map admits a tessellation representation if and only if it is essentially 2-connected. This, in particular, implies the above-mentioned result of [9] about the simultaneous upward drawings of the map $m$ and its dual $M^{*}$.

In Section 2 we present several characterizations of essentially 2-connected maps on the torus and show that they can be obtained from two minimal maps by vertex splitting and creating digons. Section 3 contains the proof of the main theorem about existence of tessellation representations. Section 4 is devoted to the visibility representation and grid contact graphs as corollaries of the main theorem. In Section 5, a characterization
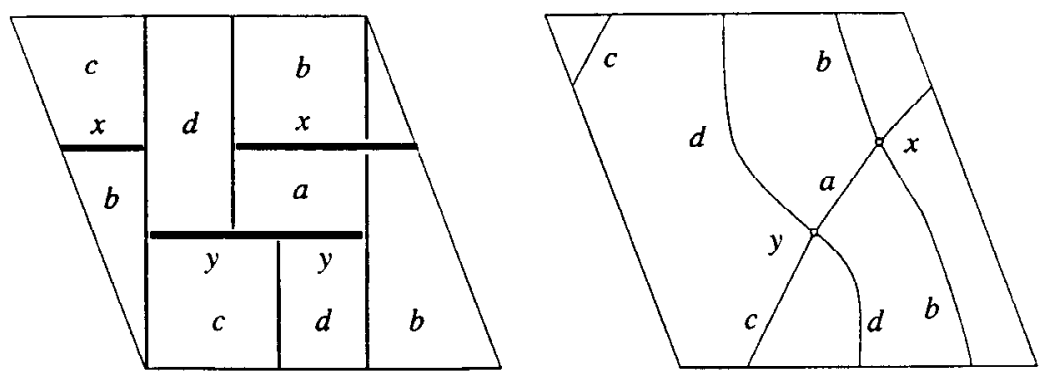

Fig. 2. A tessellation representation of a toroidal map. 


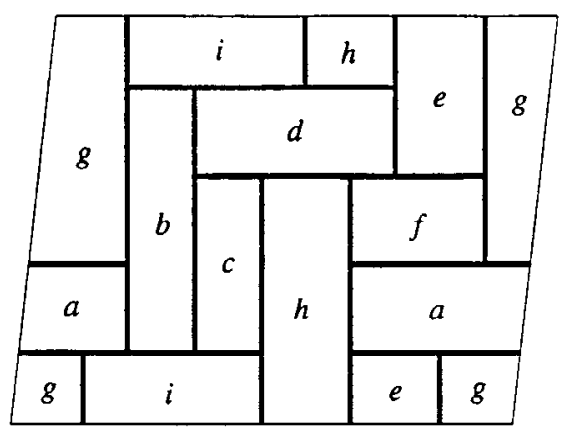

Fig. 3. A tessellation representation of the map from Fig. 1.

of those graphs that can be embedded as an essentially 2-connected map on the torus is presented.

Similar questions as addressed in this paper for the flat torus have been considered in the case of the plane by de Fraysseix et al. [3], and for the torus by Kratochvíl and Przytycka [6].

\section{Essentially 2-Connected Maps}

The universal cover of the surface $\Sigma$ is the simply connected surface $\tilde{\Sigma}$ together with a covering projection $p: \tilde{\Sigma} \rightarrow \Sigma$. We refer to [7] for the general theory of covering spaces and for basic properties of universal covering spaces. If $G$ is the graph of a map on $\Sigma$, then $p^{-1}(G)$ defines a map in $\tilde{\Sigma}$. By the homotopy lifting property of covering maps [7], a lifting of a closed walk $W$ of $G$ into the cover $\tilde{\Sigma}$ is a closed walk in $p^{-1}(G)$ if and only if $W$ is contractible in $\Sigma$. In particular, every facial walk of $G$ (i.e., the walk in $G$ corresponding to the traversal of the boundary of a face) is lifted to facial walks in the covering map.

The universal cover of the flat torus represented by the parallelogram $Q$ is the plane paved with replicates of $Q$, and the lifting of a map in the torus is an infinite plane graph. A part of such a pavement is shown in Fig. 4.

A map is essentially 2-connected if the graph of its universal cover map is 2-connected. Figure 4 shows an example (of a part) of the universal cover of an essentially 2-connected toroidal map whose graph is not 2-connected.

Lemma 2.1. Given a map $M$ of a graph $G$ on a surface $\Sigma$ whose Euler characteristic $\chi(\Sigma)$ is not positive, the following conditions are equivalent:

(i) $M$ is essentially 2-connected.

(ii) No facial walk $f$ of $M$ contains a proper closed subwalk which is contractible on $\Sigma$.

(iii) There are no planar separations in the graph $G$ of $M$, i.e., if $G_{1}$ and $G_{2}$ are graphs each having at least one edge, and such that $G=G_{1} \cup G_{2}$ and $G_{1} \cap G_{2}=\{v\}$, 


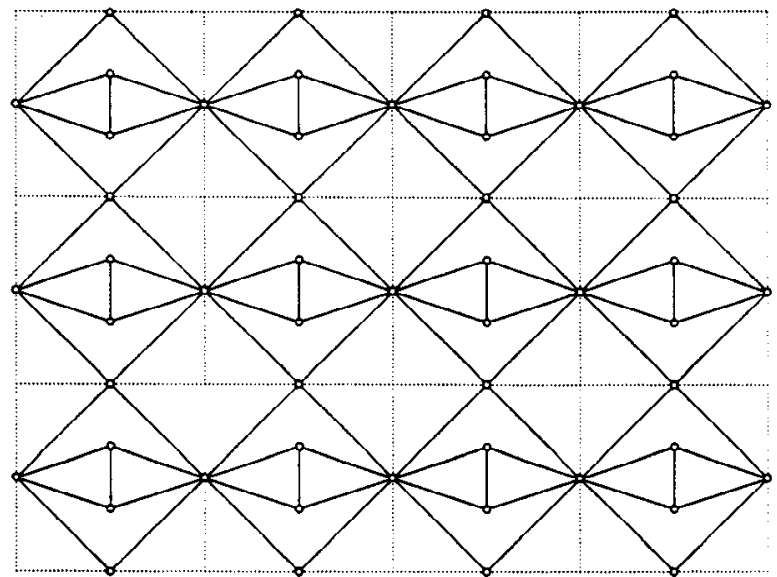

Fig. 4. A part of the universal cover of a toroidal map.

where $v$ is a vertex, then $G_{1}$ and $G_{2}$ each contain a circuit that is noncontractible on $\Sigma$.

(iv) The angle map $\mathcal{A}$ of $M$ has no homotopic pair of parallel edges, i.e., a pair of edges bounding a disk.

(v) For every subset $S$ of the vertices of the angle map $\mathcal{A}$ of $M$ we have

$$
2|S|-|E(S)| \geq 2 \chi(\Sigma) .
$$

Proof. (i) $\Leftrightarrow$ (ii) Since $\chi(\Sigma) \leq 0$, the underlying surface of the universal covering map $\tilde{M}$ of $M$ is the plane. It is well known that a (possibly infinite) graph in the plane is 2-connected if and only if every facial walk is a simple cycle (not meeting the same vertex twice). Since every facial walk (and every contractible subwalk of a facial walk) in $M$ lifts into $\tilde{M}$ to a facial walk of the same length (a subwalk of a facial walk, respectively), (i) and (ii) are easily seen to be equivalent.

(ii) $\Rightarrow$ (iii) Suppose that $G$ admits a planar separation $G=G_{1} \cup G_{2}, G_{1} \cap G_{2}=\{v\}$, and suppose that $G_{1}$ contains only circuits that are contractible on $\Sigma$. Consider a facial walk $f$ of $M$ which is not contained entirely in $E\left(G_{1}\right)$ or in $E\left(G_{2}\right)$. Then $f$ is the union of nonempty closed segments from $E\left(G_{1}\right)$ and from $E\left(G_{2}\right)$. By our choice, $G_{1}$ contains only contractible circuits. Therefore, any closed segment of $f$ in $G_{1}$ is contractible. This contradicts (ii).

(iii) $\Rightarrow$ (iv) Suppose that the angle map $\mathcal{A}$ of $M$ has a pair of parallel edges bounding an open disk $D$. Let the endpoints of these edges of $\mathcal{A}$ be $v$, a vertex of $M$, and $f$, a face of $M$. Note that $\partial \bar{D} \cap G=\{v\}$ where $\partial \bar{D}$ is the boundary of the closure of $D$ in $\Sigma$. Since $\mathcal{A}$ contains only quadrangular faces, $D$ is not a face of $\mathcal{A}$. Therefore the subgraph $G_{1}=G \cap \bar{D}$ of $G$ contains at least one edge. If $G_{2}=G \cap(\Sigma \backslash D)$, then also $G_{2}$ is nontrivial by the same reason. Since $G_{1}$ is contained in a disk, it only contains contractible circuits, so the decomposition $G=G_{1} \cup G_{2}$ is a planar separation which contradicts (iii).

(iv) $\Rightarrow$ (v) Pick $S \subseteq V(\mathcal{A})$. The angle graph $\mathcal{A}$ being bipartite, so is the subgraph 
$\mathcal{A}(S)$ of $\mathcal{A}$ induced on $S$. To prove the inequality $2|S|-|E(S)| \geq 2 \chi(\Sigma)$, it suffices to consider the case when $\mathcal{A}(S)$ is connected and when $|S| \geq 3$ since $\chi(\Sigma) \leq 0$. (Let us note that condition $\chi(\Sigma) \leq 0$ is really needed only in cases when $\mathcal{A}(S)$ is just a vertex or an edge.) Consider the induced embedding of $\mathcal{A}(S)$ in $\Sigma$. By (iv), and our assumptions on connectivity and $|S| \geq 3, \mathcal{A}(S)$ contains no digonal faces. Then it is easy to see by using the Euler's formula and the bipartiteness of $\mathcal{A}(S)$, that we get the required inequality.

(v) $\Rightarrow$ (ii) Suppose that a proper closed subwalk $Q$ of a facial walk $f$ of $M$ is contractible on $\Sigma$. Let $x$ be the endvertex of $Q \subset f$, and let $\alpha_{1}$ and $\alpha_{2}$ be the angles in $f$ corresponding to the appearances of $x$ as an end of $Q$. Let $e_{1}$ and $e_{2}$ be the edges in $\mathcal{A}$ corresponding to $\alpha_{1}$ and $\alpha_{2}$, respectively. Note that $e_{1}$ and $e_{2}$ are parallel edges, joining $x$ and $f$ in $\mathcal{A}$. They determine a closed curve $C$ on $\Sigma$ which is homotopic to $Q$ since it can be "pushed" by a homotopy within $f$ onto $Q$. Since $Q$ is contractible, so is $C$, and thus $C$ bounds an open disk $D$ in $\Sigma$. Let $S$ be the set of vertices of $\mathcal{A}$ that do not lie in $D$, and consider the corresponding submap $\mathcal{A}(S)$ of $\mathcal{A}$. Since $D$ is a disk, the underlying surface of $\mathcal{A}(S)$ is also $\Sigma$. The faces of $\mathcal{A}(S)$ coincide with faces of $\mathcal{A}$ except for the digonal face $D$. Thus $2|E(S)|$ equals four times the number of faces of $\mathcal{A}(S)$ minus 2 (due to the digon). Euler's formula then shows that $2|S|-|E(S)|=2 \chi(\Sigma)-1$. This contradicts (v).

Note that, since a map $M$ and its dual map $M^{*}$ have the same angle map, if one of $M$, $M^{*}$ is essentially 2 -connected, so is the other. The essential 2-connectivity of toroidal maps appears to be an appropriate generalization of the 2-connectivity planar maps.

Let $M$ be an essentially 2-connected map on the torus. An edge $e$ of $M$ is contractible (resp., removable), if $M / e$ (resp., $M-e$ ) is an essentially 2-connected map. Note that if $e$ is contractible (resp., removable), then its dual edge $e^{*}$ is removable (resp., contractible) in the dual map $M^{*}$.

Lemma 2.2. Let $M$ be an essentially 2-connected map on the torus and let $e \in E(M)$. Then:

(a) If e is incident with a vertex of degree 2 , then e is contractible.

(b) If $e$ is on a digonal face, then e is removable.

(c) If $M$ has no digonal faces and has at least two vertices, then M has a contractible edge.

(d) If M has no vertices of degree 2 and has at least two faces, then M has a removable edge.

Proof. Statements (a) and (b) are obvious, and (c) and (d) are dual to each other. So it suffices to prove (c). By (a) we may assume that $M$ has no vertices of degree 2.

Since $M$ has two or more vertices, there are nonloop edges in $M$. Consider $e$ being one of them. If $e$ is not contractible, its contraction results in a map that is not essentially 2-connected. According to Lemma 2.1(iv), the corresponding angle map contains a digon bounding a disk $D$. Suppose that the contracted edge $e$ was chosen in such a way that the number of vertices in $D$ is as small as possible. By our assumptions (no degree 2 vertices, no digons), $D$ contains an edge of the map $M$. This edge is then contractible 

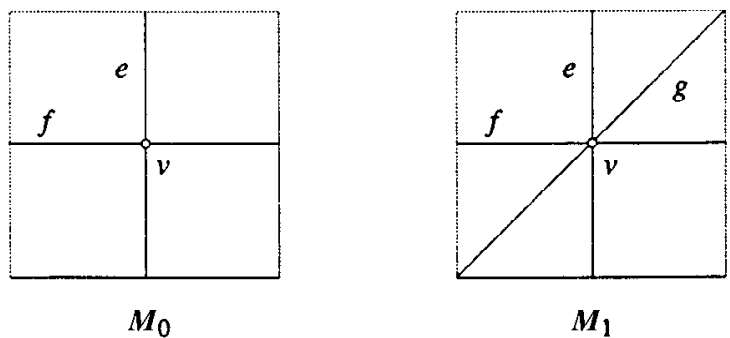

Fig. 5. Minimal essentially 2-connected maps $M_{0}$ and $M_{1}$.

with respect to $M$ since its contraction yielding a digon in the corresponding angle map would contradict the minimality of the originally chosen edge $e$ and the corresponding disk $D$.

Lemma 2.2 shows that the only minimal essentially 2-connected map (with neither contractible nor removable edges) on the torus is the map $M_{0}$ of Fig. 5. Lemma 2.2 also implies that every essentially 2-connected toroidal map $M$ can be reduced to one of the two maps $M_{0}$ and $M_{1}$ from Fig. 5 after a sequence of edge contractions and deletions of digon edges such that all intermediate maps are essentially 2-connected. Keeping track of that sequence, we may reconstruct $M$ from the map $M_{0}$ or $M_{1}$ obtained at the end by using the sequence backwards: a deletion being replaced by the addition of a homotopic parallel edge, a contraction being replaced by a vertex splitting. In other words:

Corollary 2.3. Every essentially 2-connected toroidal map can be obtained from one of the maps $M_{0}$ and $M_{1}$ by a sequence of homotopic parallel edge additions and vertex splittings such that all intermediate maps are essentially 2-connected.

\section{Tessellation Representations}

From now on we will only consider maps on the torus. In this section we will prove that a toroidal map admits a tessellation representation on the torus if and only if it is essentially 2-connected.

Suppose that we have a tessellation representation of a map $M$. This defines an orientation of edges of $M$ : each edge $e$ is oriented from the vertex corresponding to the base of the rectangle $R$ representing $e$ toward the end of $e$ corresponding to the upper side of $R$. For an arbitrary given orientation of the edges of a map $M$, an angle at a vertex $v$ is lateral if one of its arcs is incoming and the other is outgoing at $v$. Otherwise, if both arcs of an angle are incoming or both are outgoing at $v$, then the angle is extremal. Having an orientation of edges of a map $M$, we get an orientation of the angle map $\mathcal{A}$ as follows. Given an angle $\alpha$ incident with a vertex $v$ and a face $f$ of $M$, the angle $\alpha$ as an edge of $\mathcal{A}$ is oriented from $v$ to $f$ if $\alpha$ is lateral, and from $v$ to $f$ if $\alpha$ is extremal. We say that an orientation of edges of $M$ has the upward property at $v$ (resp., at $f$ ) if there are exactly two lateral angles at $v$ (resp., two extremal angles at $f$ ). Equivalently, 
in the oriented angle map $\mathcal{A}$, the indegree of every vertex (either $v$ or $f$ ) is equal to two (see Fig. 1(b)). See also [11]. An orientation of $M$, which has the upward property at every vertex and every face, is called an upward orientation. By the above remark, an upward orientation induces an indegree-two orientation of the angle map $\mathcal{A}$. (It is an easy corollary of Euler's formula that only maps with Euler characteristic zero admit upward orientations.) Having indegree-two orientation of $\mathcal{A}$, we can use the following lemma:

Lemma 3.1. Let $k$ be an integer. A graph $G$ admits an orientation of its edges such that each vertex has indegree at most $k$ if and only if the average degree of the vertices of any subgraph of $G$ is at most $2 k$.

Proof. Let $H$ be an arbitrary subgraph of $G$ and let $d^{-}(v)$ denote the indegree of $v \in V(G)$ with respect to an orientation $\Omega$ of edges of $G$. If $d^{-}(v) \leq k$ for every vertex $v$, then

$$
|E(H)| \leq \sum_{v \in V(H)} d^{-}(v) \leq k|V(H)|,
$$

so the average degree in $H$ is at most $2 k$.

Conversely, suppose that $|E(H)| \leq k|V(H)|$ for every subgraph $H$ of $G$. Let $\Omega$ be an orientation of edges of $G$ such that the number

$$
S(\Omega)=\sum_{d^{-}(v)>k}\left(d^{-}(v)-k\right)
$$

is minimal. If $S(\Omega)=0$, we are done. So, assume that $v_{0}$ is a vertex with $d^{-}\left(v_{0}\right)>k$, and let $H$ be the subgraph induced by the vertices $v$ such that there exists a directed path from $v$ to $v_{0}$. Then

$$
|E(H)|=\sum_{v \in V(H)} d^{-}(v) \leq k|V(H)|
$$

Since $d^{-}\left(v_{0}\right)>k$, there is a vertex $v_{1} \in V(H)$ such that $d^{-}\left(v_{1}\right)<k$. By reversing the arcs on a directed path from $v_{1}$ to $v_{0}$ we get an orientation $\Omega^{\prime}$ satisfying $S\left(\Omega^{\prime}\right)=S(\Omega)-1$, a contradiction.

Now we state the main result of this section.

Theorem 3.2. A toroidal map admits a tessellation representation if and only if it is essentially 2-connected.

Proof. Suppose that we have a tessellation representation of a map $M$. Then we easily get an upward drawing of $M$ which induces an upward orientation of edges of $M$. This in turn defines an orientation of edges of the angle map $\mathcal{A}$ such that every vertex of $\mathcal{A}$ 


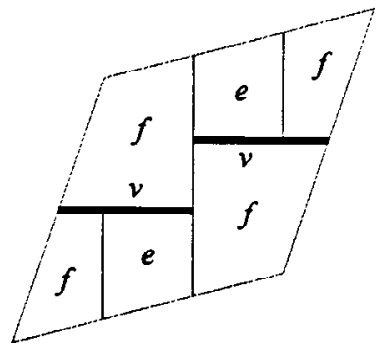

$M_{0}$

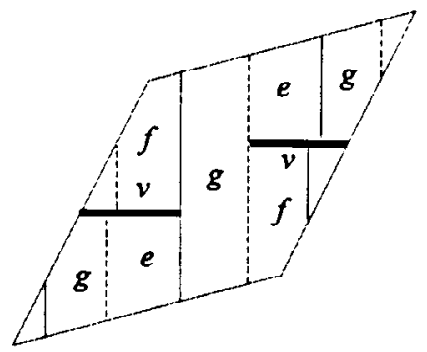

$M_{1}$

Fig. 6. Tessellation representations of maps $M_{0}$ and $M_{1}$.

has indegree exactly 2 (see the Introduction). By Lemma 3.1, every subgraph of $\mathcal{A}$ has average degree at most 4 . In our case, this means that for every subset $S$ of the vertices of $\mathcal{A}$ we have

$$
2|S|-|E(S)| \geq 0
$$

By Lemma 2.1(v), $M$ is essentially 2-connected.

To prove the converse, we have to construct a tessellation representing an arbitrary given essentially 2-connected map $M$. This is done step by step as $M$ can be obtained (according to Lemma 2.2) from $M_{0}$ and $M_{1}$ by a sequence of parallel edge additions and vertex splittings. We start with a tessellation representation of $M_{0}$ or $M_{1}$ shown in Fig. 6.

Let us now consider the two types of generating rules. Adding a parallel edge is simple: the rectangle corresponding to the edge $e$ splits into two by adding a vertical line in the middle. Of course, the new vertical segment corresponds to the digonal face.

The vertex splitting operation is slightly more involved. Up to symmetries, we have to consider four cases as displayed in Fig. 7. Note that in the first vertex-splitting rule, the orientation of the edge $e$ depends on the relative position of the two faces involved in the splitting. These rules give the procedure how to obtain a tessellation representation of $M$ starting from $M_{0}$ or $M_{1}$. The proof is complete.

The proof of Theorem 3.2 also yields a polynomial time algorithm for constructing tessellation representations of essentially 2-connected toroidal maps. Since a tessellation representation determines an upward orientation, we also get a polynomial time algorithm for upward orientations.

There are other possibilities for tessellation representations of $M_{0}$ and $M_{1}$. However, in none of them is any direction $\Delta$ or $\Delta^{\prime}$ of the tessellating rectangles parallel to the sides of the fundamental parallelogram $Q$ of the flet torus. It would be interesting to know which toroidal maps have a tessellation representation in the square model of the torus with the directions $\Delta$ and $\Delta^{\prime}$ parallel to the sides of the fundamental square. 

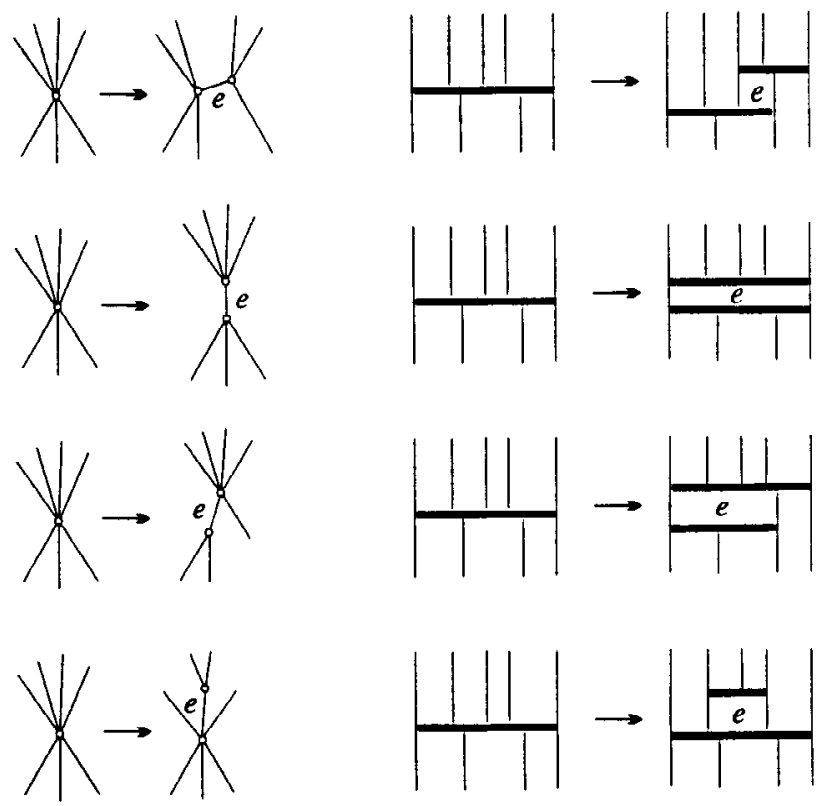

Fig. 7. Extending a tessellation after a vertex splitting.

\section{Some Corollaries on Graph Drawing}

\subsection{Upward Drawing}

A tessellation representation of a toroidal map $M$ determines an upward drawing and an upward orientation of $M$. In proving that the maps which admit tessellation representations are essentially 2-connected, we used Lemma 3.1 where only their upward orientation was needed. Therefore we also have:

Corollary 4.1. A toroidal map admits an upward orientation if and only if it is essentially 2-connected.

\subsection{Visibility Representations}

Let $M$ be an essentially 2-connected toroidal map. By Theorem 3.2 it has a tessellation representation. Let us consider the horizontal segments $H_{v}, v \in V(M)$. A vertex $v$ is adjacent to a vertex $u$ in $M$ if and only if $H_{v}$ contains a segment $S$ of positive length such that by shifting $S$ in the vertical direction $\Delta^{\prime}$ we bump into $H_{u}$ (or vice versa). We say that the segment $H_{u}$ is $\varepsilon$-visible from $H_{v}$. The intervals $H_{v}, v \in V(M)$, thus uniquely determine $M$. Such a representation of $M$ is called an $\varepsilon$-visibility representation of $M$. For the $\varepsilon$-visibility it is not important whether $H_{v}$ are open or closed segments. If we 
take as $H_{v}$ half-open segments (without their left endpoints, say), then the tessellation of $M$ yields a visibility representation for $M$ where a point of $H_{v}$ can see a point of $H_{u}$ in the vertical direction if and only if $u v \in E(M)$. Such notion of visibility (in the plane) was considered by Melnikov [8]. Tamassia and Tollis [13] and, independently, Wismath [15] characterized which graphs have such a visibility representation in the plane. Corollary 4.3 below gives a toroidal analogue of that result.

Tessellation representation is a stronger concept than $\varepsilon$-visibility representation since it gives simultaneous $\varepsilon$-visibility representations for $M$ and its dual map $M^{*}$. However, our next lemma shows that an $\varepsilon$-visibility representation of a map $M$ can be transformed into a tessellation representation of $M$.

Lemma 4.2. Every $\varepsilon$-visibility representation of a toroidal map $M$ without digonal faces can be transformed into a tessellation representation of $M$.

Proof. Let $H_{v}, v \in V(M)$, be the horizontal segments of an $\varepsilon$-visibility representation of $M$. For each edge $u v \in E(M)$, let $Q_{u v}$ be a quadrangle determined by $\varepsilon$-visibility of $H_{u}$ and $H_{v}$. The base of $Q_{u v}$ is a segment $S(u, v) \subseteq H_{u}$ (say), and the upper side of $Q_{u v}$ is a segment $S(v, u) \subseteq H_{v}$. Suppose that the quadrangles $Q_{u v}$ are chosen such that the segments $S(u, v)$ and $S(v, u)$ are maximal. Now, the vertical sides of the quadrangles $Q_{u v}, u v \in E(M)$, give rise to vertical segments in the torus. None of these segments can become a closed circle since what we started with was an $\varepsilon$-visibility representation of a 2-cell embedded graph. Now it is easy to see that we got a tessellation representation of $M$.

Theorem 3.2 and Lemma 4.2 yield:

Corollary 4.3. A toroidal map admits an $\varepsilon$-visibility representation in the flat torus (or has a visibility representation with half-open horizontal segments) if and only if it is essentially 2-connected.

\subsection{Toroidal Grid Contact Graphs}

Two families of horizontal and vertical segments of the grid of the flat torus, each one being disjoint from the others except for some contact points between two segments of different families, define a bipartite toroidal graph, called a grid contact graph. Relying on our tessellation theorem we get a characterization of the graphs which can be represented as toroidal grid contact graphs on the flat torus.

Theorem 4.4. A graph $H$ can be represented as the contact graph of straight-line segments on the flat torus where all segments corresponding to the same bipartition class of $H$ are mutually parallel if and only if $H$ is a bipartite graph that can be embedded in the torus in such a way that no pair of parallel edges bounds a disk. 
Proof. A toroidal grid contact graph representation of $H$ on the torus determines an embedding in the torus without parallel edges bounding disks. Conversely, let us embed $H$ in the torus without homotopic parallel edges. By repeatedly adding paths of length 2 or 3 between vertices of the graph it is possible to get an embedded graph $\tilde{H}$ such that:

(a) $H$ is an induced subgraph of $\tilde{H}$;

(b) $\tilde{H}$ is bipartite;

(c) $\tilde{H}$ is 2 -cell embedded (and thus also connected);

(d) $\tilde{H}$ has no homotopic parallel edges; and

(e) no face of $\tilde{H}$ has repeated vertices.

Now, beside the white bipartition class $W$ and the black class $B$ of vertices of $\tilde{H}$, consider a third class $R$ of red vertices: add a new vertex $r \in R$ in every face $f$ of $\tilde{H}$, and join $r$ by red edges to every white vertex of $f$. The resulting map $\bar{H}$ has bipartition $(W, B \cup R$ ) and also satisfies (a)-(e). By Lemma 2.1 (equivalence of conditions (i) and (iv)), $\bar{H}$ is the angle graph of an essentially 2-connected map $G$, and by Theorem 3.2 we can get a tessellation representation of $G$. It is clear that the tessellation representation yields the required grid contact representation of $\bar{H}$. By (a), the restriction to the segments corresponding to vertices of $H$ yields the required representation for $H$.

It is worth pointing out that Theorem 4.4 is closely related to results of Bellantoni et al. [2] who considered the grid dimension of graphs. By taking a parallel to results of [2], Theorem 4.4 characterizes graphs whose "toroidal grid dimension" is at most two.

\section{Graphs of Essentially 2-Connected Maps}

It is of some interest to know which abstract graphs can be obtained as graphs of essentially 2-connected toroidal maps. It is easy to find some sufficient conditions for a graph $G$ to have a representation as an essentially 2-connected toroidal map. For example, if $G$ is 2-connected, then every 2-cell embedding of $G$ in the torus yields an essentially 2-connected map. We claim that every 2-connected graph (except graphs $C_{n}, n \geq 3$ ) with genus at most 1 and has a 2-cell embedding in the torus. This is clear if the graph $G$ has genus 1 . On the other hand, if $G$ is planar, consider one of its plane embeddings. Let $e=u v$ be an arbitrary edge of $G$ with one of its endvertices, say $u$, having degree at least 3. Such an edge exists if and only if $G$ is not a cycle. Let $F_{1}, F_{2}$ be the two faces in the plane containing $e$ on the boundary. By replacing the unbounded face by a disk and adding a "handle" joining $F_{1}$ and $F_{2}$ we get an embedding of $G$ in the torus. If $f=u w \in \partial F_{1}$ is an edge at $u$ distinct from $e$, we can re-embed it by using the handle so that it attaches $u$ from "inside" of $F_{2}$. It is clear that this gives rise to a 2-cell embedding of $G$ in the torus.

Recall that a graph is nonseparable if it is either a vertex, a loop, a bond ( $p \geq 1$ parallel edges), or a 2-connected graph.

Let $G_{1}, \ldots, G_{k}(k \geq 2)$ be nonseparable graphs, each containing at least one edge and such that at least one of $G_{i}(1 \leq i \leq k)$ is not isomorphic to $K_{2}$. A graph $G$ is a cyclic amalgamation of $G_{1}, \ldots, G_{k}$ if there are vertices $u_{i}, v_{i} \in V\left(G_{i}\right)$ (possibly $u_{i}=v_{i}$ if 
$\left.G_{i} \neq K_{2}\right), i=1, \ldots, k$, and $G$ is isomorphic to the graph obtained from the (disjoint) union of $G_{1}, \ldots, G_{k}$ after identifying $u_{i}$ with $v_{i+1}$ (index modulo $k$ ), $i=1, \ldots, k$.

Theorem 5.1. A graph $G$ can be represented as an essentially 2-connected toroidal map if and only if it satisfies one of the following conditions:

(a) $G$ is 2-connected, it has an embedding in the torus, and it is not a cycle $C_{n}$ for some $n \geq 3$.

(b) $G$ is a cyclic amalgamation of nonseparable planar graphs $G_{1}, \ldots, G_{k}$ where $k \geq 2, G_{1} \neq K_{2}$, and each of $G_{1}, \ldots, G_{k}$ contains at least one edge.

Proof. We have already demonstrated sufficiency of (a). Let us now prove that graphs satisfying (b) have an essentially 2-connected toroidal representation. For $i=1, \ldots, k$ where $G_{i} \neq K_{2}$, embed $G_{i}$ in the (closed) cylinder $Q_{i}$ in such a way that $\partial Q_{i}$ is in two distinct faces, say $F_{1}, F_{2}, G_{i} \cap \partial Q_{i}=\emptyset$, and $u_{i} \in \partial F_{1}, v_{i} \in \partial F_{2}$. Such embeddings always exist. For that purpose take distinct faces $F_{1}^{\prime}, F_{2}^{\prime}$ of a planar embedding of $G_{i}$ such that $u_{i} \in \partial F_{1}^{\prime}$ and $v_{i} \in \partial F_{2}^{\prime}$. Since $G_{i}$ is nonseparable and $G_{i} \neq K_{1}, K_{2}$, such faces exist. After removing an open disk from each of $F_{1}^{\prime}$ and $F_{2}^{\prime}$, we get a required cylinder embedding. By cyclically identifying boundaries of these cylinders we get a torus embedding of $G_{1} \cup \cdots \cup G_{k}$ with vertices that need to be identified in common faces. If at least one pair $u_{i}, v_{i}$ are distinct vertices, then it is obvious that we can get a 2-cell embedding of $G$ in the torus (after adding the remaining graphs $G_{j}$ that are isomorphic to $K_{2}$, and making the appropriate identifications of vertices). If $u_{1}=v_{1}$, $u_{2}=v_{2}, \ldots, u_{k}=v_{k}$, then we get a 2-cell embedding as indicated in Fig. 8. It is easy to see by using Lemma 2.1 (ii) that the resulting maps are essentially 2 -connected.

We will now show that conditions (a) and (b) are also necessary. Let $G$ be the graph of a given essentially 2-connected map. If $G$ is 2-connected, we have (a). If $G$ is not 2connected, let $B_{0}, \ldots, B_{a}(a>1)$ be its blocks. Since the map is essentially 2-connected, each endblock $B_{i}$ contains a cycle $C_{i}$ that is noncontractible on the surface.

Suppose first that $G$ has two distinct cutvertices. Then distinct cutvertices $v, v^{\prime}$ of $G$ can be chosen such that an endblock, say $B_{1}$, contains $v$, and another endblock, say $B_{2}$, contains $v^{\prime}$. Then $C_{1}$ and $C_{2}$ are disjoint noncontractible cycles. Therefore they cut the torus into two cylinders, say $Q, Q^{\prime}$. Any other block of $G$ intersects $C_{1} \cup C_{2}$ only at $v$ or $v^{\prime}$. Since the map is 2-cell, there is a path $P \subseteq G$ in $Q$ joining $v$ and $v^{\prime}$. There is a similar path $P^{\prime}$ in $Q^{\prime}$. Let $B_{0}$ be the block of $G$ containing the cycle $C_{0}=P \cup P^{\prime}$. Now, each remaining block of $G$ is embedded entirely in $Q$ or in $Q^{\prime}$. Since each endblock $B_{i}$

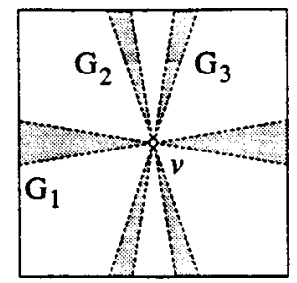

Fig. 8. The case of a single cutvertex. 
$\left(i \geq 3\right.$ ) contains a noncontractible cycle, it must intersect $C_{0}$ in a cutvertex of $G$. Now it is easy to see that $G$ fits case (b) with each of the graphs $G_{1}, \ldots, G_{k}$ being either one of $B_{1}, \ldots, B_{a}$, or a part of $B_{0}$ "between" consecutive vertices of $G$ on $P \cup P^{\prime}$ that are either cutvertices of $G$ or cutvertices of $B_{0} \cap Q$ or cutvertices of $B_{0} \cap Q^{\prime}$.

The remaining possibility is when $G$ has exactly one cutvertex $v$. Then all blocks are endblocks. If one of them contains a noncontractible cycle which does not pass through $v$, then the proof is similar to the above. Thus, we may assume that every noncontractible cycle of $G$ passes through $v$. Then $G-v$ consists of several components corresponding to the blocks of $G$. Each of such components $D$ is plane embedded (contains only contractible circuits) and all edges from $D$ to $v$ attach $D$ at the boundary of the "outer" face. Therefore all blocks of $G$ are planar graphs. Their identification at $v$ can also be interpreted as a special case of a cyclic amalgamation $\left(u_{i}=v_{i}\right.$ for every $\left.i\right)$. Hence we have (b).

It is worth mentioning that recognizing graphs satisfying (a) or (b) can be done in linear time. For (a), testing 2-connectivity is easy by a depth-first search [1], while checking if $G$ has genus at most 1 can be performed in linear time by a recent algorithm of Juvan et al. [5]. To test if $G$ satisfies (b) we first determine all blocks of $G$ and test their planarity. There must be a block $B_{0}$ containing all cutvertices (otherwise (b) is not satisfied) and such that all blocks distinct from $B_{0}$ are planar. Blocks distinct from $B_{0}$ will appear in the cyclic amalgamation with $u_{i}=v_{i}$, while $B_{0}$ itself is a cyclic amalgamation of (at least 1) planar graphs. If there are two or more cutvertices, it is easy to see how to get the corresponding decomposition. Having just one cutvertex, we simply apply the algorithm of [5].

\section{Acknowledgment}

We are greatly indebted to Jan Kratochvil and Hubert de Fraysseix for pointing out a possibility in applying Theorem 3.2 toward Theorem 4.4.

\section{References}

1. A. V. Aho, J. E. Hopcroft, and J. D. Ullman, The Design and Analysis of Computer Algorithms, AddisonWesley, Reading, MA, 1976.

2. S. Bellantoni, I. B.-A. Hartman, T. Przytycka, and S. Whitesides, Grid intersection graphs and boxicity, Discrete Math., 114 (1993), 41-49.

3. H. de Fraysseix, P. O. de Mendez, and J. Pach, Representation of planar graphs by segments, In: Intuitive Geometry (K. Boroczky and G. Fejes Toth, Eds.), North-Holland, Amsterdam, 1994, pp. 109-117.

4. J. L. Gross and T. W. Tucker, Topological Graph Theory, Wiley, New York, 1987.

5. M. Juvan, J. Marinček, and B. Mohar, Embedding a graph into the torus in linear time, submitted.

6. J. Kratochvíl and T. Przytycka, Grid intersection and box intersection graphs on surfaces (Extended Abstract), In: Graph Drawing (F. J. Brandenburg, Ed.), Springer-Verlag, Berlin, 1996, pp. 365-372.

7. W. S. Massey, Algebraic Topology: An Introduction, Harcourt, Brace, and World, New York, 1967.

8. L. A. Melnikov, Problem at the 6th Hungarian Colloquium on Combinatorics, 1981.

9. B. Mohar and P. Rosenstiehl, A flow approach to upward drawings of toroidal maps, In: Graph Drawing (R. Tamassia and I. G. Tollis, Eds.), Lecture Notes in Computer Science, Vol. 894, Springer-Verlag, New York, 1995, pp. 33-39. 
10. B. Mohar and C. Thomassen, Graphs on Surfaces, Johns Hopkins University Press, to appear.

11. P. Ossona de Mendez, Orientations bipolaires, thèse de doctorat, E.H.E.S.S., Paris, 1994.

12. P. Rosenstiehl, Embedding in the plane with orientation constraints: The angle graph, Ann. New York Acad. Sci., 1989.

13. R. Tamassia and I. G. Tollis, A unified approach to visibility representations of planar graphs, Discrete Comput. Geom. 1 (1986), 321-341.

14. R. Tamassia and I. G. Tollis, Tessellation representation of planar graphs, in Proc. Twenty-Seventh Annual Allerton Conference on Communication, Control and Computing, 1989, pp. 48-57.

15. S. K. Wismath, Characterizing bar line-of-sight graphs, Proc. ACM Symp. on Comput. Geometry, Baltimore, Maryland, 1985, pp. 147-152.

Received November 22, 1995, and in revised form May 1, 1997. 